\title{
Supraventricular tachycardia: An overview of diagnosis and management
}

\author{
Authors: Irum D Kotadia, ${ }^{A}$ Steven E Williams ${ }^{\mathrm{B}}$ and Mark O'Neill ${ }^{\mathrm{C}}$
}

\begin{abstract}
Supraventricular tachycardia (SVT) is a common cause of hospital admissions and can cause significant patient discomfort and distress. The most common SVTs include atrioventricular nodal re-entrant tachycardia, atrioventricular re-entrant tachycardia and atrial tachycardia. In many cases, the underlying mechanism can be deduced from electrocardiography during tachycardia, comparing it with sinus rhythm, and assessing the onset and offset of tachycardia. Recent European Society of Cardiology guidelines continue to advocate the use of vagal manoeuvres and adenosine as first-line therapies in the acute diagnosis and management of SVT. Alternative therapies include the use of beta-blockers and calcium channel blockers. All patients treated for SVT should be referred for a heart rhythm specialist opinion. Long-term treatment is dependent on several factors including frequency of symptoms, risk stratification, and patient preference. Management can range from conservative, if symptoms are rare and the patient is low risk, to catheter ablation which is curative in the majority of patients.
\end{abstract}

\section{Introduction}

Supraventricular tachycardia (SVT) is a heterogeneous group of arrhythmias used to describe tachycardias that involve cardiac tissue at the level of the bundle of His or above. ${ }^{1}$ The prevalence of SVT is 2.25/1000 persons with a female predominance of 2:1 across all age groups. ${ }^{2,3}$ SVT increases patient morbidity, particularly when symptoms are frequent or incessant, and in a small cohort of patients with atrial fibrillation (AF) and ventricular pre-excitation, it can be life-threatening.

This article outlines key electrocardiography (ECG) features to help narrow the differential diagnosis. Acute and long-term management strategies are then explored with specific reference to the underlying aetiology.

\footnotetext{
Authors: Aclinical research fellow, King's College London, London, UK and Guy's and St Thomas' NHS Foundation Trust, London, UK; ${ }^{B}$ Clinical lecturer, King's College London, London, UK and Guy's and St Thomas' NHS Foundation Trust, London, UK; ' Cardiology consultant and professor in cardiac electrophysiology, King's College London, London, UK and Guy's and St Thomas' NHS Foundation Trust, London, UK
}

\section{The differential diagnosis}

In the absence of left or right bundle branch block, SVTs are often referred to as narrow complex tachycardias with the most common SVTs being atrioventricular nodal re-entrant tachycardia (AVNRT), atrioventricular re-entrant tachycardia (AVRT) and atrial tachycardia (AT). Close inspection of the surface ECG to identify markers of the underlying mechanism can reveal the diagnosis in up to $80 \%$ of AVNRT or AVRT cases, although an ECG in sinus rhythm is often required for comparison (Fig 1). ${ }^{4}$

\section{Key points}

Supraventricular tachycardias (SVTs) are a common cause of presentation to primary and secondary care services and can cause significant patient discomfort and distress.

A 12-lead electrocardiography (ECG) should be performed in (and a copy given to) all patients presenting with a narrow complex tachycardia. Close inspection of the ECG, comparison with ECGs in sinus rhythm and recordings of onset/offset of tachycardia can be diagnostic.

Atrioventricular nodal re-entrant tachycardia and atrioventricular re-entrant tachycardia are re-entrant tachycardias involving the atrioventricular node and are therefore highly sensitive to termination with vagal manoeuvres and administration of adenosine.

Any SVT can present as a broad complex tachycardia due to pre-existing or rate-related bundle branch block. This can cause diagnostic uncertainty and VT must always be considered as a differential diagnosis of broad complex tachycardia.

Long-term management is dependent on underlying mechanism, frequency of symptoms and patient preference. Catheter ablation is often preferable as first-line treatment over long-term medical management in view of high curative rates and low risk of major complications.

KEYWORDS: Supraventricular tachycardia, SVT, narrow complex tachycardia, arrhythmia, ECG 
Fig 1. Diagnostic flow chart for electrocardiography in narrow complex tachycardia. $\mathrm{AF}=$ atrial fibrillation; AFlutter $=$ atrial flutter; $A T$ = atrial tachycardia; AVNRT $=$ atrioventricular nodal re-entrant tachycardia, AVRT = atrioventricular re-entrant tachycardia, VT = ventricular tachycardia.

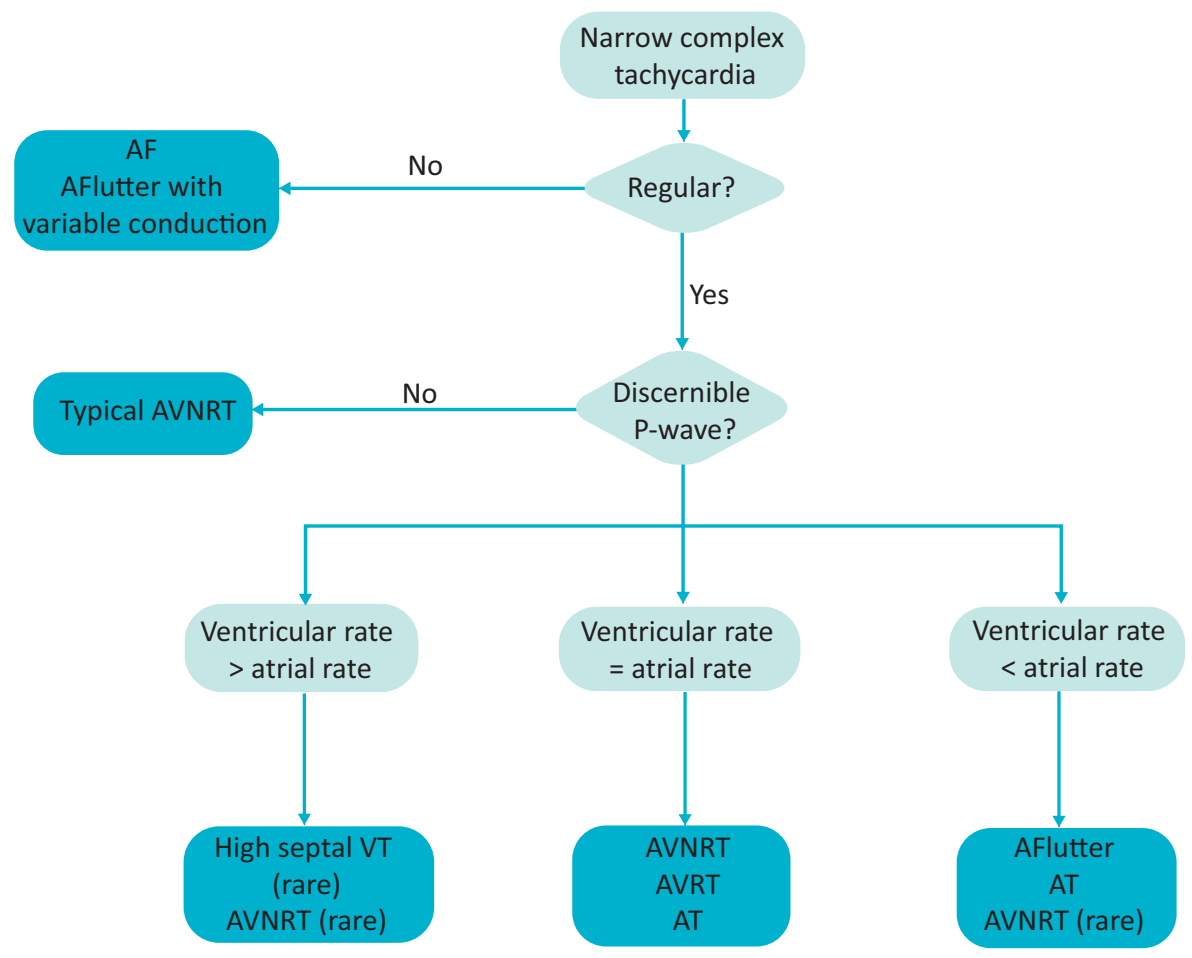

\section{Atrioventricular nodal re-entrant tachycardia}

AVNRT is the most common SVT in the general population and accounts for over $60 \%$ of patients undergoing invasive cardiac electrophysiology study. ${ }^{5}$ It is facilitated by the presence of two functionally distinct electrophysiological tracts of differing conduction velocities and refractory periods within the AV node: a fast pathway and a slow pathway. An atrial (or less commonly a ventricular) premature beat is required to initiate repetitive re-entry between the fast and slow pathways, manifest on the surface ECG as a narrow QRS tachycardia in the absence of bundle branch block.

For the most common form of AVNRT, P-waves are not easily seen on the surface ECG during tachycardia due to almost simultaneous activation of the atria and ventricles. When visible, they may be subtle, materialising as a pseudo r' wave in lead V1 or a pseudo S' deflection in the inferior leads. ${ }^{6}$ While a pseudo $r^{\prime}$ wave is more sensitive and consequently considered more valuable, previous studies have described the presence of a pseudo $S$ ' deflection to have a higher specificity and positive predictive value for AVNRT. ${ }^{4,7}$ Close inspection and comparison of the QRS complex in sinus rhythm with that during tachycardia can assist in identifying the P-wave (Fig 2).

\section{Atrioventricular re-entrant tachycardia}

In AVRT, one critical limb of the circuit is the AV node, while the other consists of an embryological remnant connecting the atria and ventricle, the accessory pathway (AP). ${ }^{8}$ These extra-nodal connections may conduct exclusively from atrium to ventricle (manifest ventricular pre-excitation or Wolff-Parkinson-White pattern ECG), from ventricle to atrium (not visible on sinus rhythm ECG and referred to as 'concealed') or be capable of conduction in both directions. Concealed APs are clinically safe as the maximum rate of ventricular activation during $A F$ remains restricted by the AV node, even in $A F^{8}{ }^{8}$

In sinus rhythm with manifest ventricular pre-excitation, both the AV node and AP may conduct to the ventricle, resulting in conduction via the AP 'pre-exciting' the ventricle ahead of conduction via the AV node and His-Purkinje tissue. This gives the characteristic ECG findings of a short PR interval ( $<120 \mathrm{~ms}$ ), slurred onset and broadening of the QRS (see Fig 2). Approximately $90 \%$ of AVRTs are orthodromic, in which the impulse travels from atrium to ventricle via the $\mathrm{AV}$ node and from ventricle to atrium via the AP. ${ }^{2}$ Retrograde P-waves are often visible after and separate from the QRS. ${ }^{9}$ The finding of electrical alternans (alternating beat variation in QRS amplitude/axis) during tachycardia is highly suggestive of AVRT, although it may also be seen in AVNRT. 7,10

Less commonly, antidromic tachycardia can occur where the AP conducts antegradely and the node provides the retrograde limb of the tachycardia as it re-enters the atrium from the ventricle via the His-Purkinje network; the QRS complex therefore appears broad and fully pre-excited. The P-waves are often difficult to see as they are buried in the ST segments and it can be difficult to differentiate antidromic AVRT from ventricular tachycardia (VT). The presence of pre-excitation during sinus rhythm recordings favours the former diagnosis.

\section{Pre-excited atrial fibrillation}

Patients with antegrade pathway conduction can be at risk of developing pre-excited atrial fibrillation. This is characterised by a broad, irregular QRS of variable duration and morphology due to conduction down both the AV node and the pathway at different rates. Rapid antegrade $\mathrm{AP}$ conduction in $\mathrm{AF}$ requires prompt clinical 
a(i)

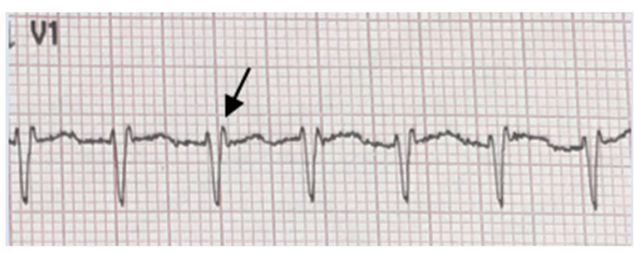

a(ii)

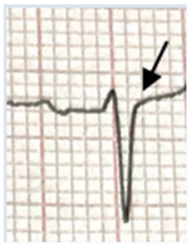

b(ii)

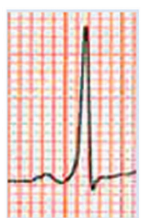

位相

b(iii)
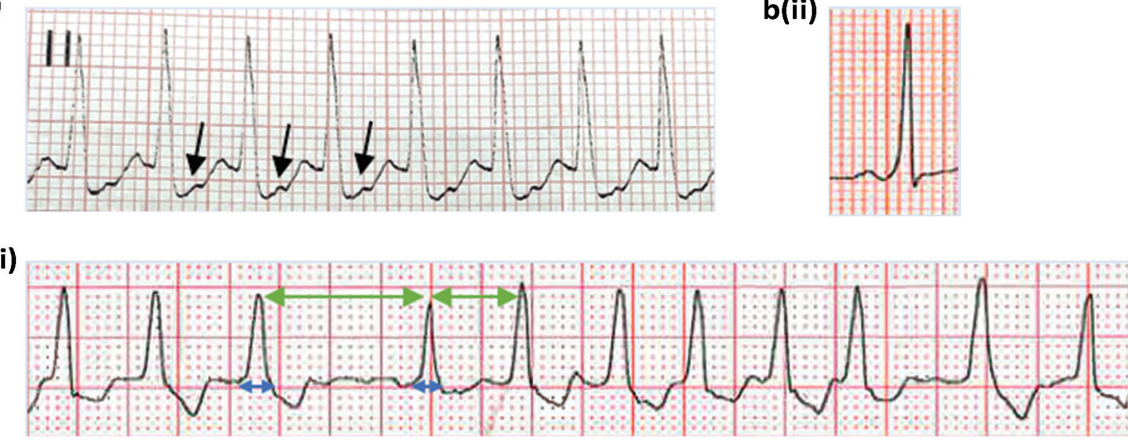

c

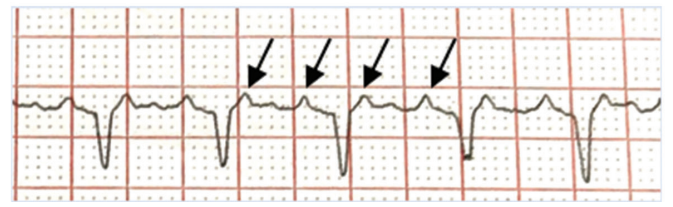

Fig 2. Characteristic electrocardiography findings in supraventricular tachycardia. a) Atrioventricular nodal re-entrant tachycardia. (i) Electrocardiography strip during tachycardia with prominent pseudo r' wave in lead V1. (ii) QRS complex from the same lead in sinus rhythm for comparison. Note the absence of the pseudo r' wave present in tachycardia. b) Atrioventricular re-entrant tachycardia. (i) Narrow complex tachycardia with visible retrograde $P$-waves that appear separate to the QRS. (ii) Sinus rhythm snapshot showing the characteristic short PR interval, slurred upstroke and broad QRS complex. (iii) Pre-excited atrial fibrillation. Note the irregular rhythm (green arrows) and the variable QRS duration (blue arrows). This is a medical emergency. c) Atrial tachycardia. Narrow complex tachycardia with monomorphic P-waves and stable cycle length. attention and an electrophysiology opinion should be sought early due to the risk of ventricular fibrillation if incorrectly managed. ${ }^{3}$

\section{Atrial tachycardia}

Atrial tachycardia (AT) originates within the atrium and is oblivious to the behaviour of the AV node. On surface ECG, the P-waves appear monomorphic with a stable tachycardia cycle length, but morphologically distinct from the sinus P-wave. There are two mechanisms - focal and re-entrant - with typical atrial flutter being by far the most common AT seen in clinical practice. In all forms of AT, the ventricular rate is dependent on the conduction properties of the AV node; atrial flutter is not commonly associated with 1:1 atrioventricular conduction. Focal AT (non-re-entrant) may show rate variability due to acceleration and deceleration while atrial flutter is a fixed atrial (P-wave) rate tachycardia with variability seen in the ventricular (QRS) rate according to the behaviour of the AV node. ${ }^{11}$

\section{Acute management of SVT}

Acute management of a regular narrow complex tachycardia entails modification of the arrhythmia circuit leading to termination while simultaneously reaching a diagnosis where possible. A 12-lead ECG is critical to establish diagnosis and guide both acute and long-term specialist management.

\section{Vagal manoeuvres}

If the patient is haemodynamically stable, vagal manoeuvres can be attempted in the first instance. For the Valsalva manoeuvre, SVT termination rates vary dramatically from $19 \%$ to $54 \%$, with the REVERT trial demonstrating a significant improvement when performed in the semi-recumbent position. ${ }^{2,12}$ The manoeuvre can be reproduced by instructing the patient to blow into a $10 \mathrm{~mL}$ syringe with enough force to move the plunger. If carotid sinus massage is being performed, the patient's neck must be extended and turned away. The carotid sinus can then be located behind the angle of the jaw. Firm pressure should be applied unilaterally for no longer than 5 seconds and the manoeuvre avoided in patients with known carotid artery disease, previous transient ischaemic attack / stroke and carotid bruits. ${ }^{1,3}$ This can be performed in the lying and sitting position and on both sides.

\section{Adenosine}

If unsuccessful, and depending on the clinical setting, an adenosine challenge can be administered via a large peripheral/ central vein as a bolus immediately followed by a $10 \mathrm{~mL}$ rapid saline flush. Delivery to the myocardium must be swift as the half-life of adenosine is less than 10 seconds. Adenosine acts directly on the $A V$ node $G_{i}$-coupled $A_{1}$ receptors, hyperpolarising the cell membrane, slowing and usually inhibiting AV nodal conduction. ${ }^{13}$ This is a crucial diagnostic manoeuvre and a continuous ECG / rhythm strip should be recorded during administration. One of three responses may occur (Fig 3). If no change is seen, either the dose/delivery of adenosine was inadequate or rarely, the diagnosis is VT in which the AV node plays no part. A sudden termination is suggestive of AVNRT or AVRT as the AV node is a critical part of the circuit in both; the mean dose of adenosine required for termination is $6 \mathrm{mg}$. The presence of P-waves with AV dissociation is diagnostic of AT; termination of AT with adenosine is unusual but favours a focal over a re-entrant mechanism. ${ }^{14}$ 
a

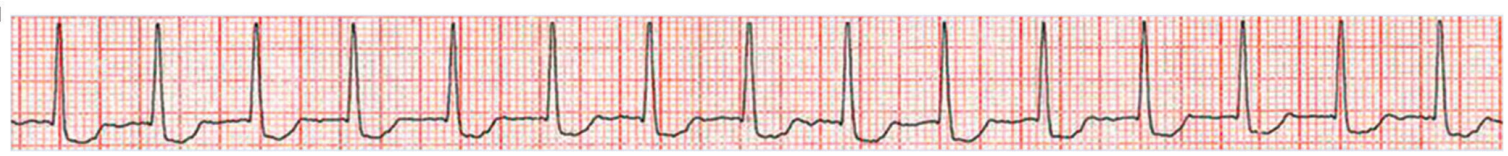

b

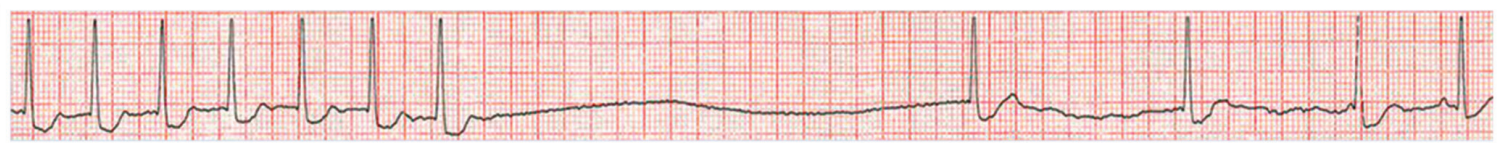

C n

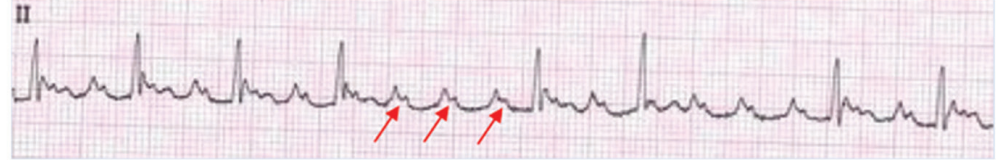

Fig 3. Response to adenosine challenge. a) Inadequate delivery. There is no evidence of conduction/block in the atrioventricular node. This is highly suggestive of ineffective administration of adenosine. Rarely this can be indicative of high septal VT which may present with a narrow complex tachycardia. b) Atrioventricular block and termination of tachycardia achieved. The first three beats following termination are of junctional origin. The final beat shows restoration of sinus rhythm once the adenosine has been metabolised. c) Persistent tachycardia with slowing of ventricular response. Atrioventricular delay/block. P-waves continue to 'march' through despite atrioventricular block (arrows). Tachycardia continues with recovery of ventricular response rate following metabolism of adenosine.

If there is evidence of haemodynamic compromise, the patient should undergo immediate direct current cardioversion, however adenosine can be trialled while preparations are underway. Importantly, adenosine may induce atrial fibrillation and electrical cardioversion should be available where ventricular pre-excitation is suspected. ${ }^{15}$ European Society of Cardiology guidelines advocate cautious use in asthmatics and verapamil as an alternative only in those with severe asthma. ${ }^{2}$ See Fig 4 for overview of management of SVT. ${ }^{2}$

\begin{tabular}{|c|c|c|}
\hline Drug & Initial IV dose & Further dosing if unsuccessful \\
\hline Adenosine & $6 \mathrm{mg}$ stat & $\begin{array}{l}\text { Further } 12 \mathrm{mg} \text { after } 1-2 \text { mins } \\
\text { (max } 18 \mathrm{mg} \text { stat) }\end{array}$ \\
\hline Verapamil & $\begin{array}{l}5-10 \mathrm{mg} \\
\text { over } 5 \text { mins }\end{array}$ & Further $5-10 \mathrm{mg}$ after 5 mins \\
\hline Diltiazem & $\begin{array}{l}0.25 \mathrm{mg} / \mathrm{kg} \\
\text { over } 2 \mathrm{mins}\end{array}$ & $\begin{array}{l}\text { Further } 0.35 \mathrm{mg} / \mathrm{kg} \text { after } 15 \mathrm{mins} \\
\text { can be given in AF/AFlutter in } \\
\text { heart rate }>100 \mathrm{bpm}\end{array}$ \\
\hline Meto & $\begin{array}{l}1-2 \mathrm{mg} / \mathrm{min} \text {, } \\
\text { up to } 5 \mathrm{mgl}\end{array}$ & $\begin{array}{l}\text { Further } 5 \mathrm{mg} \text { after } 5 \text { mins } \\
\text { (max } 10-15 \mathrm{mg} \text { ) }\end{array}$ \\
\hline
\end{tabular}

\section{Supraventricular} tachycardia<smiles>C1=[Te]C2CCCCC2C1</smiles>

Haemodynamically
stable? $\quad$ No $\longrightarrow \begin{gathered}\text { Synchronised } \\ \text { cardioversion }\end{gathered}$
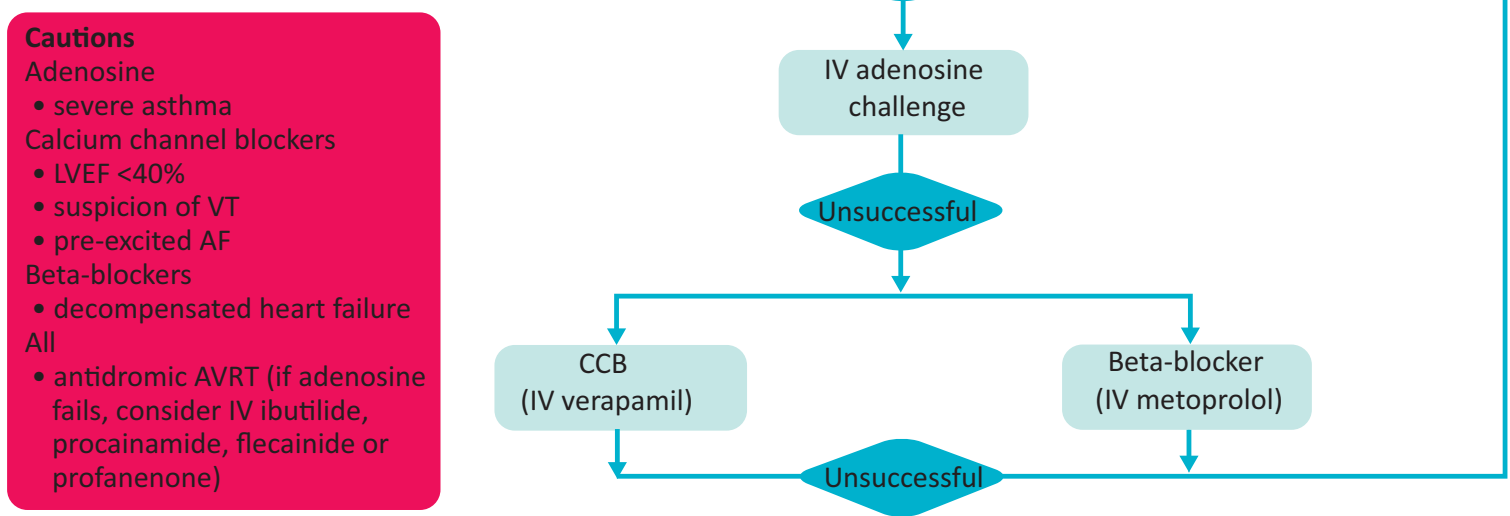

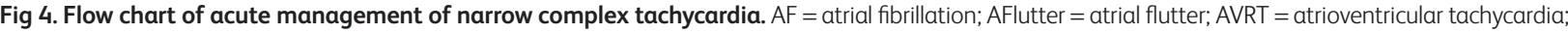
$\mathrm{bpm}=$ beats per minute; $\mathrm{CCB}=$ calcium channel blocker; $\mathrm{IV}=$ intravenous; $\mathrm{LVEF}=$ left ventricular ejection fraction; VT = ventricular tachycardia. 


\section{Calcium channel blockers and beta-blockers}

If adenosine is unsuccessful or contraindicated, calcium channel blockers (diltiazem and verapamil) and beta-blockers (metoprolol) can be particularly useful in slowing AV node conduction acutely. ${ }^{2}$ Beta-blockers are less efficacious at terminating the arrhythmia, whereas a clinical trial comparing medical termination of SVT showed comparable success rates with graded administration of adenosine (up to $12 \mathrm{mg}$ ) and high-dose intravenous verapamil $(7.5 \mathrm{mg}) .{ }^{16}$ More recently, a trial of intranasal etripamil (shortacting calcium channel blocker, currently in phase II clinical trials) terminated supraventricular arrhythmias in $65-95 \%$ of cases. ${ }^{2,17}$ Both drug groups should be used cautiously in patients with decompensated heart failure and hypotension. Concomitant use should be avoided where possible due to the risk of significant hypotension and bradycardia. Calcium channel blockers should also be avoided in patients with evidence of left ventricular dysfunction (ejection fraction $<40 \%$ ) or any suspicion of $\mathrm{VT} .{ }^{2} \mathrm{AV}$ node blocking drugs are absolutely contraindicated in pre-excited atrial fibrillation.

\section{Long-term management}

Long-term management is contingent on the underlying mechanism, frequency of symptoms, patient safety and preference. However, the potential for substantial improvements in quality of life, reduced hospital attendances and cost burden make catheter ablation a particularly desirable option as first line therapy for all SVTs, especially in AVNRT and AVRT where documented cure rates can exceed $95 \%$ with an associated risk of $<1 \%$ for major complications. ${ }^{2}$

In AVRT, where a manifest pathway is present and symptoms are infrequent, it is important to assess the risk of sudden cardiac death. Continuous ambulatory monitoring or an exercise test may show intermittent or sudden loss of pre-excitation suggestive of a low-risk pathway. ${ }^{18}$ If this is not present an invasive cardiac electrophysiology study may be required to characterise electrophysiological properties of the pathway for risk stratification. Catheter ablation is strongly recommended in the event of a high-risk pathway being diagnosed.

\section{Conclusion}

Supraventricular tachycardia is a common cause of hospital attendance and acute admission. With close inspection of the ECG in sinus rhythm at the time of onset, offset and during tachycardia, a diagnosis can often be formulated. This can guide the method and urgency of treatment required, as well as allow initial patient counselling for long-term management.

\section{References}

1 Katritsis DG, Boriani G, Cosio FG et al. European Heart Rhythm Association (EHRA) consensus document on the management of supraventricular arrhythmias, endorsed by Heart Rhythm Society (HRS), Asia-Pacific Heart Rhythm Society (APHRS), and Sociedad Latinoamericana de Estimulación Cardiaca y Elect. Eur Heart ] 2018;39:1442-5.
2 Brugada J, Katritsis DG, Arbelo E et al. 2019 ESC guidelines for the management of patients with supraventricular tachycardia. The Task Force for the management of patients with supraventricular tachycardia of the European Society of Cardiology (ESC). Eur Heart J 2019:ehz467.

3 Lee KW, Badhwar N, Scheinman MM. Supraventricular tachycardiapart I. Curr Probl Cardiol 2008:33:467-546.

4 Kalbfleisch SJ, El-Atassi R, Calkins H, Langberg J], Morady F. Differentiation of paroxysmal narrow QRS complex tachycardias using the 12-lead electrocardiogram. J Am Coll Cardiol 1993;21:85-9.

5 Orejarena LA, Vidaillet H, Destefano F et al. Paroxysmal supraventricular tachycardia in the general population. J Am Coll Cardiol 1998:31:150-7.

6 Katritsis DG, Camm AJ. Classification and differential diagnosis of atrioventricular nodal re-entrant tachycardia. Europace 2006;8: 29-36.

7 Erdinler I, Okmen E, Oguz E et al. Differentiation of narrow QRS complex tachycardia types using the 12-lead electrocardiogram. Ann Noninvasive Electrocardiol 2002;7:120-6.

8 Delacrétaz E. Clinical practice. Supraventricular tachycardia. N Engl J Med 2006:354:1039-51.

9 Katritsis DG, Josephson ME. Differential diagnosis of regular, narrow-QRS tachycardias. Hear Rhythm 2015;12:1667-76.

10 Wellens $\mathrm{HJ}$. The value of the ECG in the diagnosis of supraventricular tachycardias. Eur Heart J 1996;17(Suppl C):10-20.

11 Roberts-Thomson KC, Kistler PM, Kalman JM. Focal atrial tachycardia I: clinical features, diagnosis, mechanisms, and anatomic location. Pacing Clin Electrophysiol 2006;29:643-52.

12 Appelboam A, Reuben A, Mann C et al. Postural modification to the standard Valsalva manoeuvre for emergency treatment of supraventricular tachycardias (REVERT): a randomised controlled trial. Lancet 2015;386:1747-53.

13 Rankin AC, Oldroyd KG, Chong E, Rae AP, Cobbe SM. Value and limitations of adenosine in the diagnosis and treatment of narrow and broad complex tachycardias. $\mathrm{Br}$ Heart J 1989;62:195-203.

14 Glatter KA, Cheng J, Dorostkar P et al. Electrophysiologic effects of adenosine in patients with supraventricular tachycardia. Circulation 1999:99:1034-40.

15 Turley AJ, Murray S, Thambyrajah J. Pre-excited atrial fibrillation triggered by intravenous adenosine: a commonly used drug with potentially life-threatening adverse effects. Emerg Med J 2008:25:46-8.

16 Nath S, DiMarco JP, Haines DE. Basic aspects of radiofrequency catheter ablation. J Cardiovasc Electrophysiol 1994;5:863-76.

17 Stambler BS, Dorian P, Sager PT et al. Etripamil nasal spray for rapid conversion of supraventricular tachycardia to sinus rhythm. J Am Coll Cardiol 2018;72:489-97.

18 Sharma AD, Yee R, Guiraudon G, Klein GJ. Sensitivity and specificity of invasive and noninvasive testing for risk of sudden death in Wolff-Parkinson-White syndrome. J Am Coll Cardiol 1987;10:373-81.

Address for correspondence: Dr Irum D Kotadia, School of Biomedical Engineering and Imaging Sciences, King's College London, 4th Floor North Wing, St Thomas' Hospital, Westminster Bridge Road, London SE1 7EH, UK. Email: irum.kotadia@kcl.ac.uk 